TRANSACTIONS OF THE

AMERICAN MATHEMATICAL SOCIETY

Volume 359, Number 3, March 2007, Pages 1253-1263

S 0002-9947(06)04279-6

Article electronically published on October 17, 2006

\title{
ON LORENTZ DYNAMICS: FROM GROUP ACTIONS TO WARPED PRODUCTS VIA HOMOGENEOUS SPACES
}

\author{
A. AROUCHE, M. DEFFAF, AND A. ZEGHIB
}

\begin{abstract}
We show a geometric rigidity of isometric actions of non-compact (semisimple) Lie groups on Lorentz manifolds. Namely, we show that the manifold has a warped product structure of a Lorentz manifold with constant curvature by a Riemannian manifold.
\end{abstract}

\section{INTRODUCTION}

Recall the following result of [10, which shows how homogeneous spaces are rare in Lorentz geometry (in comparison with the Riemannian case, say).

Theorem $1.1([10])$. If $(M, g)$ is a homogeneous Lorentz space of dimension $\geq 3$, with irreducible isotropy group, then it has constant sectional curvature.

Observe that the statement in [10] seems weaker than that above, since the isotropy group is assumed to satisfy the supplementary condition of non-precompactness. However, this follows from irreducibility. Indeed, in the same vein as [10], the principal result of [3] says how irreducibility is strong in the Lorentz setting.

Theorem $1.2([3])$. A Lie subgroup $H$ (not assumed a priori to be closed) of $O(1, n)$, which does not preserve any one-dimensional isotropic subspace of $\mathbb{R}^{1+n}$, is up to conjugacy, a union of some components of some $O(1, p) \subset O(1, n)$. In particular, if $H$ acts irreducibly on $\mathbb{R}^{1+n}$, then $H$ contains $S O_{0}(1, n)$.

Our goal in the present article is to relax homogeneity by considering (nontransitive) isometric group actions. This work is actually motivated by the study of isometric Lie group actions on non-compact Lorentz manifolds, for instance in the same vein as [1, 7, 6, ,...

To simplify, we will always assume that all the given group actions are faithful.

1.1. Warped product structure versus partial homogeneity. We first ask whether there is an adaptation of Theorem 1.1 to non-transitive isometric actions. In this situation, we consider a group $G$ acting isometrically on a Lorentz manifold $(M, g)$. Each orbit is a homogeneous space. However, the causal type of the orbit may be timelike, spacelike, or lightlike, that is, the induced metric may be Lorentzian, Riemannian or degenerate, respectively. The following generalization of Theorem 1.1 relies on the existence of orbits of Lorentz type satisfying irreducibility. It says roughly that the space is partially of constant curvature.

Received by the editors December 14, 2004.

2000 Mathematics Subject Classification. Primary 53C50, 54H15.

Key words and phrases. Lorentz manifolds, warped product, semi-simple transformation groups.

(C)2006 American Mathematical Society 
Theorem 1.3. Let $G$ be a Lie group acting isometrically on a Lorentz manifold $(M, g)$ of dimension $\geq 3$. Suppose there exists an orbit $N$ which is a (homogeneous) Lorentz space with irreducible isotropy (restricted to TN).

Then $N$ is a complete space of constant (sectional) curvature, and $G$ contains the identity component I $\operatorname{som}^{0}(N)$ as a factor.

Furthermore, a neighborhood of $N$ is a warped product $L \times_{w} N$, where $L$ is some Riemannian manifold and the factor $N$ corresponds to the orbits of $I_{\operatorname{som}}^{0}(N)$.

Definition and fundamental properties of warped products are in $₫ 2$,

1.2. Non-properness versus irreducibility. Let us go a step further, and try to get rid of the irreducibility hypothesis. In fact, irreducibility is an algebraic condition which looks somehow inappropriate in our dynamic-geometric setting here. We want to substitute for it a more natural dynamical condition. Our theory is that non-properness is good enough for this role.

1.2.1. Recalls. We find it worthwhile to "make some order" around the concept of non-properness of actions. This will be useful in the sequel (statements and proofs).

Recall that an action of a group $G$ on a space $M$ is called proper if, for any sequences $\left(x_{n}\right)$ of $M$, and $\left(g_{n}\right)$ of $G$, whenever $\left(x_{n}\right)$ and $\left(g_{n} x_{n}\right)$ converge in $M$, then some subsequence of $\left(g_{n}\right)$ converges in $G$.

For our purpose here the following variant will be useful. If $M$ is a metric space (this is the case for a manifold, since it can be endowed with a Riemannian metric), a family $\mathcal{F}$ of mappings is said to be equicontinuous on a point $x \in M$ if for each $\epsilon>0$ there exists $\eta>0$ such that for $y \in M$ and $f \in \mathcal{F}$ we have $d(x, y)<\eta \Rightarrow d(f(x), f(y))<\epsilon$. The family is said to be equicontinuous on a subset $U \subseteq M$ if it is equicontinuous on every point in $U$. We say that the action of $G$ is locally equicontinuous, if for any sequences $\left(x_{n}\right)$ of $M$, and $\left(g_{n}\right)$ of $G$, whenever $\left(x_{n}\right)$ and $\left(g_{n} x_{n}\right)$ converge in $M$, then a subsequence of $\left(g_{n}\right)$ is equicontinuous on a neighborhood of the limit $x$ of $\left(x_{n}\right)$. Therefore, a subsequence of $\left(g_{n}\right)$ is converging in the group of homeomorphisms of that neighborhood, but the limit is not necessarily a restriction of an element of $G$. Obviously, a nonlocally equicontinuous action is non-proper. The converse is not true. The standard example of a non-proper but locally equicontinuous action is the usual linear action of $\mathbb{R}$ on the torus with dense orbits. In general, the any non-closed Lie group of the isometry group of a compact Riemannian manifold is locally equicontinuous but not proper. Another example is the action of the universal cover $\tilde{G}$ on a Lie group $G$ (via the canonical projection). It is always locally equicontinuous, but proper only if $G$ has a finite fundamental group (because it is not faithful). Observe nevertheless:

Fact 1.4. Let $G$ be a Lie group acting by preserving a pseudo-Riemannian structure on a manifold $M$. If $G$ is the full isometry group, or if $G$ is semi-simple with finite center, then its action is non-proper iff it is non-equicontinuous.

For the proof, recall the well-known fact that a $C^{0}$-limit of pseudo-Riemannian (smooth) isometries is a smooth isometry, and that the Lie group topology coincides with the $C^{0}$ topology. This is equivalent to saying that the isometry group is closed in the group of homeomorphisms. (Actually, this fact is true for all rigid geometric structures.) For $G$ a simple Lie group with finite center, recall that its image under a homomorphism into any Lie group is closed, and that $G$ is a finite cover of it. An analogous argument applies to the semi-simple case with finite center. 
Therefore, in statements (essentially within proofs) below that involve semisimple Lie groups, we will not worry about distinction between compactness and pre-compactness.

A $G$-homogeneous space $G / H$ is non-proper if the left $G$-action on it is nonproper. This is equivalent, for general $G$, to non-precompactness of $H$ and to non-compactness of $H$ when $G$ is semi-simple.

1.2.2. Semi-simple group actions with non-proper orbits. Without an a priori irreducibility hypothesis, we have the following generalization of Theorem 1.3, assuming the orbits are non-proper and the group $G$ is semi-simple (a kind of intrinsic irreducibility).

Theorem 1.5. Let $G$ be a connected semi-simple Lie group acting isometrically on a Lorentz manifold $(M, g)$ of dimension $\geq 3$. Suppose that no (local) factor of $G$ is locally isomorphic to $S L(2, \mathbb{R})$ and that there exists a non-proper orbit $N$ of Lorentz type (that is, $N$ has non-compact isotropy).

Then, up to a finite cover, $G$ factors $G=G_{2} \times G_{1}$, where:

- $G_{1}$ possesses an orbit $N_{1}$ which is a Lorentz space of constant (non-vanishing) curvature, and $G_{1}$ equals Isom $^{0}\left(N_{1}\right)$.

- There is a G-invariant neighborhood $U$ of $N$ which is a warped product $L \times{ }_{w} N_{1}$.

- The factor $N_{1}$ corresponds to $G_{1}$-orbits, and $G_{2}$ acts along the L-factor.

The following fact will be useful in proofs, and also gives an exact geometric and algebraic description of $N, N_{1}, G, G_{1}, \ldots$

Proposition 1.6. Let $G$ be a connected Lie group acting isometrically and transitively on a Lorentz curvature space $N$ of constant curvature.

1) Assume the isotropy group of $G$ is irreducible. Then, $G$ equals $\operatorname{Isom}^{0}(N)$, and $N$ is one of the following:

i) The Minkowski flat space.

ii) The de Sitter space $S O(1, n+1) / S O(1, n)$ (of positive constant curvature).

iii) $A$ (cyclic) covering of the anti de Sitter space $S O(2, n) / S O(1, n)$ (of negative constant curvature).

2) Assume $G$ is semi-simple, and the isotropy is non-compact. Then the same conclusion holds but the Minkowki possibility is excluded.

Remarks 1.7.

1) In both theorems above, the warped product is local, i.e. not the whole space is a warped product. To see this, one considers the $O(1, n)$-action on the Minkowski space $\mathbb{R}^{1, n}$. If the Lorentz quadratic form is $q=-x_{0}^{2}+x_{1}^{2}+\ldots+x_{n}^{2}$, then the warped product is defined exactly on the region $q>0$.

2) The result does not seem to be optimal, that is, it might be generalized to other groups.

3) Warped product structures on universal covers of compact Lorentz manifolds with strong dynamics are obtained, for instance, in [4, 11, 12].

1.3. From non-proper actions to non-proper homogeneous spaces. Let us go another step, by asking how to get such non-proper orbits from a global condition on the action. For instance, is orbital non-properness inherited from non-properness of the (ambient) action?

Theorem 1.8. Let $G$ be a semi-simple Lie group of finite center acting isometrically and non-properly on a Lorentz manifold $M$. Suppose that no (local) factor 
of $G$ is locally isomorphic to $S L(2, \mathbb{R})$. Then there is a point with a non-compact stabilizer. In particular, the restriction of the action of $G$ to its orbit is nonproper. More precisely, the stabilizer of some point contains a non-trivial unipotent one-parameter group. (In other words, a non-proper Lorentz G-space contains a non-proper $G$-homogeneous orbit.)

This result allows one to get from (non-transitive) actions to homogeneous (i.e., transitive) ones. This is a common philosophy for actions with strong dynamics in a geometric setting. The result here is in particular reminiscent of the so-called Zimmer Embedding Theorem (see for instance [13]). Unfortunately, the orbit is a non-proper homogeneous space, but not necessarily Lorentz! The nuisance is that it can be lightlike (degenerate); another story.

\section{Proof of Theorem 1.3}

\subsection{An algebraic lemma.}

Lemma 2.1. Let $E$ (resp. F) be a Lorentz (resp. Euclidean) vector space. Denote by $O(E)$ and $O(F)$ their respective orthogonal groups. Let $H^{\prime}$ be a Lie subgroup of $O(E) \times O(F)$, whose projection on $O(E)$ acts irreducibly on $E$.

Then, $H^{\prime}$ contains a subgroup $H \subset O(E) \times\{1\}$, which contains the identity component of $O(E) \times\{1\}$. In particular:

- Any linear $H$-invariant mapping $f: E \rightarrow F(f \circ h=f$, for any $h \in H)$ is trivial.

- The same is true for any $H$-invariant bilinear antisymmetric mapping $E \times E \rightarrow$ $F$.

Proof. We infer from the irreducible case of Theorem 1.2 that the projection $H$ of $H^{\prime}$ on $O(E)$ contains the identity component of $O(E)$ (isomorphic to $O(1, n)$ for $1+n=\operatorname{dim} E)$, say $H=O(E)$, to simplify notation.

Since $O(F)$ is compact, $H$ is isomorphic to the non-compact semi-simple Levi factor of $H^{\prime}$. Therefore, $H^{\prime}$ contains a subgroup isomorphic to $H$, that is, there exists a homomorphism $\rho: H=O(E) \rightarrow O(F)$, such that the graph $\{(h, \rho(h)), h \in$ $O(E)\}$ is contained in $H^{\prime}$.

Now, $\rho$ must be trivial since a semi-simple Lie group of non-compact type has no non-trivial homomorphism into a compact group.

For the last two conclusions of the lemma, one can assume $F=\mathbb{R}$. The kernel of the linear mapping $f$ is $O(E)$-invariant; hence, it is trivial by irreducibility. A similar argument yields triviality of invariant antisymmetric bilinear mappings.

\subsection{Group actions.}

2.2.1. Proof of the first part of Proposition 1.6. From Theorem 1.1 and the irreducibility hypothesis, $N$ has constant curvature. Let us consider the case where $N$ is flat; the proof is the same in the other cases. Thus, $N$ is locally isometric to the Minkowski space $\mathbb{R}^{1, n}$, for which the identity component of the isometry group is the semi-direct product $S O(1, n) \ltimes \mathbb{R}^{1+n}$. From Theorem 1.2, the stabilizer in $G$ must equal $S O(1, n)$ (we assumed $G$ connected). It follows that $G$ intersects the translation group $\mathbb{R}^{1+n}$ in an open subgroup, and therefore, $G$ contains all translations, and hence equals $S O(1, n) \ltimes \mathbb{R}^{1+n}$, and $N$ is exactly the Minkowski space. (Another way to conclude would be to observe that the full isometry group of the 
Minkowski space is generated by the stabilizers of its points; in fact, just stabilizers of two different points generate.)

Lemma 2.2. Let $G$ be a connected Lie group acting isometrically on a Lorentz manifold $(M, g)$. Let $N$ be an orbit of $G$, which is of Lorentz type and has an irreducible stabilizer (when acting on the tangent space of $N$ ).

Then $G$ splits $G=K \times G_{1}$, where $G_{1}=I_{\operatorname{som}^{0}}(N)$, and $K$ acts trivially on $N$ ( $K$ is in fact precompact in the stabilizer of any point of $N$ ).

Furthermore, in a neighborhood of $N$, all the orbits of $G_{1}$ are isometric, and thus determine a foliation. The orthogonal distribution to this foliation is integrable, and all structures are invariant under the $G$-action.

Proof. The group $G$ acts on $N$ via a homomorphism $G \rightarrow I_{s o m}^{0}(N)$. Its image $G_{1}$ has irreducible isotropy, and therefore, from the first part of Proposition 1.6, $N$ has constant curvature and $G_{1}=\operatorname{Isom}^{0}(N)$.

We now check that, in fact, $G_{1}$ is contained in $G$, and hence $G$ splits as claimed. For this, consider $x_{0} \in N$, and denote by $H^{\prime}$ its isotropy group. The orthogonal space $L_{x_{0}}$ of $T_{x_{0}} N$ in $T_{x_{0}} M$ is spacelike (the metric on it is positive definite). We are in a position to apply Lemma 2.1 with $E=T_{x_{0}} N$ and $F=L_{x_{0}}$. It then follows that the identity component of the isotropy group of $x_{0}$ in $G_{1}$ is contained in $H^{\prime}$, and in particular in $G$. However, $G_{1}$ is generated by stabilizers of various points of $N$, and therefore, $G_{1}$ is contained in $G$.

Next, we investigate the $G_{1}$-action on $M$ near $N$. Let $H$ be the isotropy group of $x_{0}$ in $G_{1}$. Its action on $L_{x_{0}}$ is trivial. Let $\exp _{x_{0}}$ denote the exponential mapping for the Lorentz metric and consider the (local) submanifold $\mathcal{L}_{x_{0}}=\exp _{x_{0}}\left(L_{x_{0}}\right)$. Then $\exp _{x_{0}}$ conjugates the infinitesimal action of $H$ on $L_{x_{0}}$ with its action on $\mathcal{L}_{x_{0}}$. In particular, $H$ acts trivially on this latter submanifold. That is, $H$ is contained in the isotropy group of any point of $\mathcal{L}_{x_{0}}$. An obvious semi-continuity argument implies that isotropy groups cannot be bigger. Therefore, we have a foliation by $G_{1}$-orbits, all satisfying the same irreducibility condition for their isotropy groups. Let us denote this foliation by $\mathcal{N}$ and its tangent bundle by $T \mathcal{N}$. Let $L$ be the orthogonal distribution. The obstruction to integrability of $L$ can be measured by means of a tensor $T: L \times L \rightarrow T \mathcal{N}$. It is defined by $T(X, Y)$, which equals the orthogonal projection on $T \mathcal{N}$ of the bracket $[X, Y]$, where $X$ and $Y$ are sections of $L$. Since the isotropy group acts trivially on $L$ and irreducibly on $T \mathcal{N}, T$ is trivial, that is, $L$ is integrable.

2.3. Warped product, end of the proof. Let $(L, h)$ and $(N, m)$ be two pseudoRiemannian manifolds and $w: L \rightarrow \mathbb{R}^{+}-\{0\}$ a warping function. The warped product $M=L \times{ }_{w} N$, is the topological product $L \times N$, endowed with the pseudoRiemannian metric $g=h \oplus w m$.

Our goal now is to prove that an open neighborhood of $N$ in $M$ is a warped product. So far, we have the orthogonal foliations $\mathcal{N}$ and $\mathcal{L}$. One can say that the De Rham decomposition theorem is a criterion for a pair of such foliations to determine a (local) direct pseudo-Riemannian product. The condition is that (the tangent bundles of) $\mathcal{N}$ and $\mathcal{L}$ are parallel, or a priori more weakly, that leaves of $\mathcal{N}$ and $\mathcal{L}$ are geodesic. There is a similar, but more complicated, criterion for warped products [5, 8. We will not use this criterion, but rather give a brief proof in our case. Our terminology here is close to that of [12, which may be consulted for a more complete exposition. Let $N$ and $L$ be (local) leaves of a point $x_{0}$ for the 
foliations $\mathcal{N}$ and $\mathcal{L}$, respectively. So, locally, $M$ has an adapted topological product $L \times N$. The metric can be written

$$
g_{(l, n)}=h_{(l, n)} \oplus m_{(l, n)} .
$$

- Let us show that $h_{(l, n)}=h$, that is, it does not depend on $n$. This is clear since $G_{1}$ acts isometrically: if $k \in G_{1}$, then it sends $\mathcal{L}_{(l, n)}$ to $\mathcal{L}_{k(l, n)}$, where $k(l, n)$ has the form $\left(l, n^{\prime}\right)$ (orbits of $G_{1}$ correspond to $\mathcal{N}$ ). Therefore $g=h \oplus m_{(l, n)}$ (the geometric meaning of this fact is that $\mathcal{N}$ is a geodesic foliation [12]).

- In order to understand the variation of $m_{(l, n)}$ as a function of $(l, n)$, write $x_{0}=\left(l_{0}, n_{0}\right)$, fix $l_{1} \in L$ and consider the mapping

$$
S:\left(l_{0}, n\right) \in N=\mathcal{N}_{\left(l_{0}, n_{0}\right)} \rightarrow\left(l_{1}, n\right) \in \mathcal{N}_{\left(l_{1}, n_{0}\right)} .
$$

$S$ commutes with the $G_{1}$-action on the $G_{1}$-orbits of $\left(l_{0}, n_{0}\right)$ and $\left(l_{1}, n_{0}\right)$. In particular it commutes with the isotropy actions at these two points. As shown previously these isotropy groups are the full orthogonal groups of the Lorentz scalar products on their tangent spaces. In particular, they preserve, up to a multiplicative constant, only one Lorentz scalar product. This means that $S$ is a homothety at $\left(l_{0}, n_{0}\right)$ : the image metric equals the metric at $\left(l_{1}, n_{0}\right)$ (along $\left.\mathcal{N}_{\left(l_{1}, n_{0}\right)}\right)$ up to a multiplicative factor $w\left(l_{0}, n_{0}\right)$. Now, since $S$ commutes with the (full) action on orbits, it follows that $w$ does not depend of $n$. That is, if $m=m_{\left(l_{0}, n\right)}$ is the metric on $N$, then $m_{(l, n)}=w(l) m$. In sum, $g=h \oplus w(l) m$, that is, $M$ is a warped product.

- The fact that $N$ has constant curvature follows from Theorem 1.1 since $N$ has irreducible isotropy.

- Finally, the splitting of $G$ is that given in Lemma 2.2, which is obviously compatible with the warped product structure.

\section{Proof of Theorem 1.5}

We will first prove Theorem 1.5 under an additional homogeneity assumption, that is, $G$ acts transitively on $M$. This will be a generalization of Theorem 1.1 . where one keeps the non-precompactness assumption and replaces the irreducibility one by the semi-simplicity of the ambient group. We will the come back to the proof of Theorem 1.5 by the end of the present section, after proving the following transitive particular case.

Theorem 3.1. Let $(M, g)$ be a G-homogeneous Lorentz space of dimension $\geq 3$, with non-precompact isotropy group, and $G$ a connected semi-simple Lie group with no (local) factor locally isomorphic to $S L(2, \mathbb{R})$.

Then, there is a group direct product $G=G_{2} \times G_{1}$, and a metric direct product $M=L \times N$, where $L$ is a Riemannian $G_{2}$-homogeneous manifold, $N$ is a Lorentz space of constant non-vanishing curvature, and $G_{1}=\operatorname{Isom}^{0}(N)$.

Proof. For $x \in M$, and a lightlike (i.e. isotropic) vector $u \in T_{x} M$, consider the orthogonal hyperplane $u^{\perp}$. Let $C_{x}$ be the set of those $u$ for which $u^{\perp}$ is tangent to a totally geodesic (lightlike) hypersurface, that is, $\exp _{x}\left(u^{\perp}\right)$ is a totally geodesic hypersurface (near $x$ ). The crucial fact, proved in [10, is that non-precompactness of the isotropy group $H_{x}$ implies $C_{x}$ is non-empty.

- Suppose $C_{x}$ is finite. One can (locally) define only finitely many continuous sections $x \rightarrow u(x) \in C_{x}$. In particular, one can suppose that these sections are invariant under the $G$-action. In fact, to simplify notation in the following argument, one is allowed to suppose that $C_{x}$ has (everywhere) cardinality 1 . Therefore, 
we have a $G$-invariant distribution of hyperplanes $x \rightarrow u(x)^{\perp}$. This distribution is integrable, the leaf at $x$ being the geodesic hypersurface

$$
\mathcal{H}_{u}=\exp _{x}\left(u^{\perp}\right) .
$$

From this we get that $M$ possesses a codimension one $G$-invariant foliation. The quotient space is a 1-manifold. But a simple Lie group acting (non-trivially) on a 1 -manifold must be locally isomorphic to $S L(2, \mathbb{R})$. This is impossible because of our assumption on $G$.

It then follows that $C_{x}$ is infinite, and hence generates a Lorentz subspace $F_{x}$ of dimension $\geq 3$.

- The same argument as above allows one to check that the isotropy group cannot preserve a space of dimension 1 . Nor can it preserve a Lorentz subspace of dimension 2, since the two isotropic directions in such a subspace would be preserved individually. To summarize, one can select a subspace $E_{x}$ of $F_{x}$ which is Lorentz, generated by its infinite subset $E_{x} \cap C_{x}$, and on which the isotropy group acts irreducibly.

- From this, we define a $G$-invariant distribution $E$ on which the isotropy group, at each point, acts irreducibly. As in the proof of Theorem 1.3, one defines an integrability obstruction tensor for $E$, which must vanish, by Lemma 2.1. since it is antisymmetric and invariant under the isotropy group. Therefore $E$ is integrable. We denote by $\mathcal{N}$ its tangent foliation.

- Let $N$ be a leaf of $\mathcal{N}$, and $G_{3}$ the subgroup of $G$ leaving it invariant. This subgroup acts transitively on $N$, since the foliation $\mathcal{N}$ is invariant under the transitive $G$-action. Moreover, $G_{3}$ contains the stabilizers (in $G$ ) of all the points of $N$, which, by construction, act irreducibly on the tangent space of each point of $N$. We can thus apply Theorem 1.3 to the $G_{3}$-action. In particular, $N$ has constant curvature, and $G_{3}$ contains $G_{1}=I_{s o m}^{0}(N)$, which induces a warped product $L \times_{w} N$ in a neighborhood of $N$ in $M$.

- One shows in a standard way the coincidence of the foliation $\mathcal{N}$ (given by the distribution $E$ ) and that given by the factor $N$ in the warped product. Therefore, the warped product is $G$ invariant, and is in particular global: $M=L \times{ }_{w} N$.

- Now, we argue by contradiction to show that $N$ has non-vanishing curvature, that is, $N$ is not the Minkowski space. Indeed, $N$ can be identified with the quotient space $M / \mathcal{L}$ endowed with its similarity Lorentz structure (that is, a Lorentz metric up to a (global) constant), which is preserved by the $G$-action. In other words, the semi-simple group $G$ acts transitively by homothety on $N$. However, the similarity group of the Minkowski space $\mathbb{R}^{1, n}$ is $\left(\mathbb{R}^{+} \times O(1, n)\right) \ltimes \mathbb{R}^{1+n}$. Any semi-simple subgroup of it is conjugate into $O(1, n)$, and cannot act transitively.

- Since $G$ acts transitively on $M=L \times_{w} N$ by preserving the warped product structure, all the leaves $\{l\} \times N$ are isometric, and hence have the same curvature. However, metrics at two levels $l_{1}$ and $l_{2}$ are related by a factor $\frac{w\left(l_{1}\right)}{w\left(l_{2}\right)}$. Curvatures are related by the inverse ratio. From constancy of the (non-vanishing) curvature, we infer that $w$ is a constant function, that is, $M=L \times N$ is a direct product.

- Since $G$ preserves the product structure of $M$ and contains $G_{2}$, which is the full (identity component of the) isometry group of $N, G$ splits as claimed. This finishes the proof of Theorem 3.1 .

3.0.1. Proof of the last part of Proposition 1.6. A direct algebraic proof is surely available. This is also an easy corollary of Theorem 3.1. Indeed, if $M$ has constant 
curvature, then, it cannot have a non-trivial decomposition $M=L \times N$. Therefore, $M=N$, that is, $G=\operatorname{Isom}^{0}(M)=\operatorname{Isom}^{0}(N)$.

3.0.2. End of the proof of Theorem 1.5. The splitting of $G$ is that given by Theorem 3.1. We apply Theorem 1.3 to the $G_{1}$-action in order to obtain a warped product as claimed.

Remark 3.2. A particular case of Theorem 3.1 is where $G$ is simple. It can be reformulated in this case as follows: if a simple group $G$ acts by preserving a Lorentz metric on a quotient $G / H$, where $H$ is non-compact, then this quotient has constant curvature, that is, $G / H$ has the form $O(1, n+1) / O(1, n)$ or $O(2, n) / O(1, n)$ (up to a cyclic cover). This was proved in [9] under the a priori assumption that $G$ is (up to a cover) $O(1, n+1)$ or $O(2, n)$.

\section{Proof of Theorem 1.8}

The following method has become a standard ingredient in the study of "geometric" $G$-actions; see for instance [1, 2, 7, 6, .... One considers the action of the group $G$ on the space $S^{2}(\mathcal{G})$ of symmetric bilinear forms on its Lie algebra $\mathcal{G}$. There is a $G$-equivariant Gauss map $\Phi: M \rightarrow S^{2}(\mathcal{G})$. Non-properness of the $G$-action on $M$ translates to a non-properness of the action of $G$ on the image $\Phi(M)$. This latter action is "algebraic", it has a poor dynamics, and is easy to understand. From this, one hopes to get information about the $G$-action on $M$.

In our case here, one shows that there exists a point $q \in M$ such that $\mathcal{G}$ admits an isotropic subspace with respect to the symmetric bilinear form $\Phi(q)$, of dimension $\geq 2$. Then the non-precompactness of the stabilizer $\operatorname{stab}(q)$ follows.

4.1. The Gauss map. Let $G$ be a Lie group acting by isometries on a Lorentz manifold $(M, h)$. For each $X \in \mathcal{G}$, let $\bar{X}$ be the vector field on $M$ given by

$$
\bar{X}_{x}=\left.\frac{d}{d t}(\exp (t X) \cdot x)\right|_{t=0} .
$$

Let $\Phi: M \rightarrow S^{2}(\mathcal{G})$ be the so-called Gauss map given by

$$
x \mapsto \Phi_{x}:(X, Y) \mapsto h_{x}\left(\overline{X_{x}}, \overline{Y_{x}}\right) .
$$

Recall the definition of the $G$-action on $S^{2}(\mathcal{G})$ given by

$$
(g . q)\left(X_{1}, X_{2}\right)=q\left(A d_{g^{-1}} X_{1}, A d_{g^{-1}} X_{2}\right)
$$

for $q$ in $S^{2}(\mathcal{G})$ and $g$ in $G$, and $X_{1}, X_{2} \in \mathcal{G}$.

Then $\Phi$ is equivariant, that is,

$$
g . \Phi_{x}=\Phi_{g \cdot x} \forall g \in G .
$$

Indeed, for $g \in G$ and $X \in \mathcal{G}$, we have

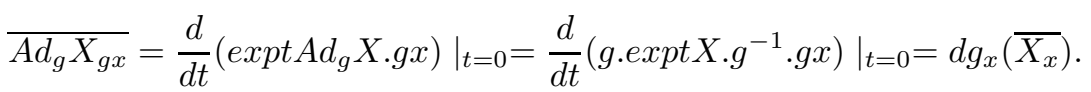

Hence, for $X, Y \in \mathcal{G}, x \in M$ and $g \in G$, we get (using the fact that $G$ acts on $M$ by isometries)

$$
\begin{gathered}
g . \Phi_{x}(X, Y)=h_{x}\left({\overline{A d_{g^{-1}} X}}_{x},{\overline{A d_{g^{-1}} Y}}_{x}\right)=h_{x}\left(d g_{g x}^{-1} \bar{X}_{g x}, d g_{g x}^{-1} \bar{Y}_{g x}\right) \\
=h_{g x}\left(\bar{X}_{g x}, \bar{Y}_{g x}\right) \\
=\Phi_{g x}(X, Y) .
\end{gathered}
$$


Observe that if $G$ acts non-properly on $M$, then it does so on $\Phi(M)$.

4.2. Root decomposition. Let $\mathcal{A}$ be a Cartan subalgebra, that is, a maximal abelian $\mathbb{R}$-split subalgebra of $\mathcal{G}$ and $A$ the associated Cartan group. Let $\Psi=$ $\Psi(\mathcal{A}, \mathcal{G})$ be the root system of $(\mathcal{A}, \mathcal{G})$ and

$$
\mathcal{G}=\mathcal{G}_{0} \oplus \bigoplus_{\alpha \in \Psi} \mathcal{G}_{\alpha}
$$

the root space decomposition where

$$
\begin{gathered}
\mathcal{G}_{\alpha}=\{X \in \mathcal{G}: \text { adH.X }=\alpha(H) . X, \forall H \in \mathcal{A}\}, \\
\mathcal{G}_{0}=\{X \in \mathcal{G}: \text { adH.X }=0, \forall H \in \mathcal{A}\} .
\end{gathered}
$$

Then $A$ acts on $\mathcal{G}$ by diagonal matrices, since

$$
A d_{g^{-1}}=A d_{e x p H}=e^{a d H}=\operatorname{diag}\left(e^{\alpha(H)}\right)_{\alpha \in \Psi \cup\{0\}},
$$

where $g^{-1}=\exp (H), H \in \mathcal{A}$. It follows that $A$ acts by diagonal matrices on $S^{2}(\mathcal{G})$, and this latter representation admits the following decomposition:

$$
S^{2}(\mathcal{G})=\bigoplus_{\lambda \in \Psi \cup\{0\}+\Psi \cup\{0\}} V_{\lambda},
$$

where $V_{\lambda}$ is the set of symmetric bilinear forms $q$ on $\mathcal{G}$ satisfying

$$
q\left(\exp (H) \cdot X_{1}, \exp (H) \cdot X_{2}\right)=e^{\lambda(H)} \cdot q\left(X_{1}, X_{2}\right),
$$

for all $H \in \mathcal{A}$ and all $X_{1}, X_{2} \in \mathcal{G}$. Keeping in mind that for $X_{1} \in \mathcal{G}_{\alpha}$ and $X_{2} \in \mathcal{G}_{\beta}$ we have

$$
q\left(\exp (H) \cdot X_{1}, \exp (H) \cdot X_{2}\right)=e^{(\alpha+\beta)(H)} \cdot q\left(X_{1}, X_{2}\right) .
$$

It follows that the forms $q$ in $V_{\lambda}$ satisfy :

$$
\alpha+\beta \neq \lambda \Rightarrow \mathcal{G}_{\alpha} \perp \mathcal{G}_{\beta}
$$

4.3. Properness of abelian actions. The following is a criterion for the nonproperness of linear actions of abelian Lie groups

Lemma 4.1. Let $\left\{\lambda_{1}, \cdots, \lambda_{n}\right\}$ be a generating system in $\mathbb{R}^{d}$. Let $\mathbb{R}^{d}$ act faithfully on $\mathbb{R}^{n}$ by diagonal matrices as follows. For $t \in \mathbb{R}^{d}$, set $M(t)=\operatorname{diag}\left(e^{\left\langle\lambda_{i}, t\right\rangle}\right)_{1 \leq i \leq n}$, where $\langle.,$.$\rangle is the usual inner product in \mathbb{R}^{d}$. Assume $V$ is an invariant (topological) subspace of $\mathbb{R}^{n}$ on which the action is non-proper. Then there exists a non-zero vector $t_{0} \in \mathbb{R}^{d}$ and either an element $x \in \mathbb{R}^{n}$ such that $x_{i}=0$ if $\lambda_{i}\left(t_{0}\right)<0$ or an element $y \in \mathbb{R}^{n}$ such that $y_{i}=0$ if $\lambda_{i}\left(t_{0}\right)>0$.

Proof. Since the action on $V$ is non-proper, there exists a sequence $\left(t_{p}\right)$ with $t_{p} \rightarrow$ $+\infty$ in $\mathbb{R}^{d}$ and a sequence $\left(x_{p}\right)$ in $V$ such that $x_{p} \rightarrow x$ in $V$ and $y_{p}=t_{p} . x_{p} \rightarrow y$ in $V$. Consider the sequence $\frac{t_{p}}{\left\|t_{p}\right\|}$. Up to taking a subsequence, we may assume it has a limit $t_{0}$. Since the action is faithful, and $t_{0} \neq 0$, there exists $i_{0} \in\{1, \cdots, d\}$ such that $\lambda_{i_{0}}\left(t_{0}\right) \neq 0$. Note that $\lambda_{i}\left(t_{p}\right) \rightarrow+\infty$ if $\lambda_{i}\left(t_{0}\right)>0$ and $\lambda_{i}\left(t_{p}\right) \rightarrow-\infty$ if $\lambda_{i}\left(t_{0}\right)<0$. Hence $x^{i}=0$ if $\lambda_{i}\left(t_{0}\right)>0$ and $y^{i}=0$ if $\lambda_{i}\left(t_{0}\right)<0$. 
4.4. End of the proof. As we mentioned above, $G$ acts non-properly on $\Phi(M)$. Let $G=K A K$ be the Cartan decomposition of $G$. Since $G$ has a finite center, $K$ is compact. So $A$ acts also nonproperly on $\Phi(M)$. From this, it follows that there exists $H \neq 0, q \in \Phi(M)$ and $\lambda_{0} \in \Psi \cup\{0\}+\Psi \cup\{0\}$ such that $\lambda_{0}(H)<0$ and $q_{\lambda}=0$ for all $\lambda \in \Psi \cup\{0\}+\Psi \cup\{0\}$ with $\lambda(H)<0$. Put $q=\Phi_{x}$. Then $\bigoplus_{\alpha(H)<0} \mathcal{G}_{\alpha}$ is isotropic with respect to $\Phi_{x}$. Hence the image of $\bigoplus_{\alpha(H)<0} \mathcal{G}_{\alpha}$ by the map $X \mapsto \bar{X}_{x}$ is an isotropic subspace of $T_{x} M$, so its dimension is less than or equal to 1 . However:

Fact 4.2. For any $H \in \mathcal{A}$, the dimension of $\bigoplus_{\alpha(H)<0} \mathcal{G}_{\alpha}$ is at least 2 (where $G$ is assumed to have no local factor locally isomorphic to $S L(2, \mathbb{R})$ ).

Proof. This dimension cannot be 0 , since $G$ is semi-simple. If it equals 1 , then the subalgebra $\bigoplus_{\alpha(H) \geq 0} \mathcal{G}_{\alpha}$ has codimension 1 in $\mathcal{G}$. This contradicts the nonexistence of an $S L(2, \mathbb{R})$ factor (only simple groups locally isomorphic to $S L(2, \mathbb{R}$ ) act on 1-manifolds).

We infer from this the existence of a non-zero element $X \in \bigoplus_{\alpha(H)<0} \mathcal{G}_{\alpha}$ such that $\bar{X}_{x}=0$, which yields $\exp (t X) \in \operatorname{stab}(x), \forall t \in \mathbb{R}$. But elements of $\bigoplus_{\alpha(H)<0} \mathcal{G}_{\alpha}$ are nilpotent, and thus generate non-compact groups. This finishes the proof of Theorem 1.8 ,

\section{ACKNOWLEDGMENT}

We would like to thank the referee for his remarks and suggestions, especially for allowing us to fix the statement of Theorem 1.3. This work has been partially supported by the CNRS and the ACI "Structures géométriques et trous noirs".

\section{REFERENCES}

[1] S. Adams, Dynamics of simple Lie groups on Lorentz manifolds. Geom. Dedicata 105 (2004), 1-12. MR2057240 (2005d:37050)

[2] S. Adams, G. Stuck, The isometry group of a compact Lorentz manifold. I, II. Invent. Math. 129 (1997), no. 2, 239-261, 263-287. MR1465326 (98i:53092)

[3] C. Boubel, A. Zeghib, Isometric actions of Lie subgroups of the Moebius group. Nonlinearity 17 (2004), no. 5, 1677-1688. MR2086144 (2005e:37049)

[4] M. Gromov, Rigid transformations groups. Géométrie différentielle (Paris, 1986), 65-139, Travaux en Cours, 33, Hermann, Paris, 1988. MR0955852 (90d:58173)

[5] S. Hiepko, Eine innere Kennzeichnung der verzerrten Produkte. Math. Ann. 241 (1979) 209215. MR0535555 (81a:53037)

[6] N. Kowalsky, Actions of non-compact simple groups of Lorentz manifolds. C. R. Acad. Sci. Paris Sér. I Math. 321 (1995), no. 5, 595-599. MR1356560 (96g:53093)

[7] N. Kowalsky, Noncompact simple automorphism groups of Lorentz manifolds. Ann. Math. 144 (1997), 611-640. MR1426887 (98g:57059)

[8] R. Ponge; H. Reckziegel, Twisted products in pseudo-Riemannian geometry. Geom. Dedicata 48 (1993), no. 1, 15-25. MR1245571 (94h:53093)

[9] D. Witte, Homogeneous Lorentz manifolds with simple isometry group. Beiträge Algebra Geom. 42, no. 2 (2001) 451-461. MR1865533 (2002i:53064)

[10] A. Zeghib, Remarks on Lorentz symmetric spaces, Compositio Math. 140 (2004) 1675-1678. MR2098408 (2005g:53127)

[11] A. Zeghib, Sur les espaces-temps homogènes. The Epstein birthday schrift, 551-576, Geom. Topol. Monogr., 1, Geom. Topol. Publ., Coventry, 1998. MR.1668344 (99k:57079) 
[12] A. Zeghib, Isometry groups and geodesic foliations of Lorentz manifolds. Part II: Geometry of analytic Lorentz manifolds with large isometry groups. GAFA, 9 (1999) 823-854. MR:1719610 (2001g:53060)

[13] R. Zimmer, On the automorphism group of a compact Lorentz manifold and other geometric manifolds. Invent. Math. 83 (1986) 411-426. MR0827360 (87j:58019)

Faculté des Mathématiques, Université des Sciences et de la Technologie Houari Boumediene, BP 32 El'Alia, Bab Ezzouar, Alger, Algeria

E-mail address: arouche@math.usthb.dz

Faculté des Mathématiques, Université des Sciences et de la Technologie Houari Boumediene, BP 32 El'Alia, Bab Ezzouar, Alger, Algeria

E-mail address: deffaf1@yahoo.fr

CNRS, UMPA, École Normale Supérieure de Lyon, 46, allée d'Italie, 69364 Lyon CEDEX 07, France

E-mail address: Zeghib@umpa.ens-lyon.fr

$U R L$ : www .umpa.ens-lyon.fr/ ₹eghib/ 